\title{
Japanese Dairy Cattle Productivity Analysis using Bayesian Network Model (BNM)
}

\author{
Iqbal Ahmed \\ Graduate School of Science and \\ Engineering \\ Saga University, Saga, Japan
}

Kenji Endo

Morinaga Dairy Service Co. Ltd.

1-159 Toyoharaotsu, Nasugun

Nasumachi,

Tochigi 329-3224, Japan

\author{
Osamu Fukuda \\ Graduate School of Science and \\ Engineering \\ Saga University, Saga, Japan
}

\author{
Kohei Arai \\ Graduate School of Science and \\ Engineering \\ Saga University, Japan
}

\author{
Hiroshi Okumura \\ Graduate School of Science and \\ Engineering \\ Saga University, Japan
}

\begin{abstract}
Kenichi Yamashita
Advanced Manufacturing Research Institute, The National Institute of Advanced Industrial Science and Technology(AIST), Tosu 841-0052, Japan
\end{abstract}

\begin{abstract}
Japanese Dairy Cattle Productivity Analysis is carried out based on Bayesian Network Model (BNM). Through the experiment with 280 Japanese anestrus Holstein dairy cow, it is found that the estimation for finding out the presence of estrous cycle using BNM represents almost 55\% accuracy while considering all samples. On the contrary, almost $73 \%$ accurate estimation could be achieved while using suspended likelihood in sample datasets. Moreover, while the proposed BNM model has more confidence than the estimation accuracy lies in between 93 to $100 \%$. In addition, this research also reveals the optimum factors to find out the presence of estrous cycle among the 270 individual dairy cows. The objective estimation methods using BNM definitely lead a unique idea to overcome the error of subjective estimation of having estrous cycle among these Japanese dairy cattle.
\end{abstract}

Keywords-Bayesian Network Model; BCS; Postpartum Interval; Parity Number; Estrous Cycle; Cattle Productivity

\section{INTRODUCTION}

Dairy cattle productivity largely depends on pure and more accurate understanding of the presence of estrous cycle. The subjective methods of finding estrous cycle in cows, such as ultrasound image analysis by an experienced inspector could jeopardize the farm productivity. The Bayesian Network Model (BNM) with the inclusion of Body Condition Parameter (BCS), Postpartum Interval (PPI) and Parity number could be used to overcome the error in subjective estimation of estrous cycle presence in cows. This research reveals that the approach of using BNM with other parameters, exhibit more objectively accurate estimation of the presence of estrous cycle in the industry and thereby, helping to the farm management to design proper estrous synchronization protocol. The Body Condition Score: BCS, Postpartum Interval: PPI and Parity Number have taken into consideration for designing the proposed BNM.

The paper describes research background first followed by introducing of BNM. Then experimental procedure and results is described followed by conclusion with some discussions.

\section{RESEARCH BACKGROUND}

A sound understanding of the presence or absence of estrous cycle allows cattle producers to troubleshoot reproductive problems in their farms. These understanding are also important when using estrous synchronization and other reproductive technologies in dairy industry. The estrous cycle of cattle is the period from one estrus (heat, phase of sexual receptivity) to the next estrus. For the cow and heifer, this period averages 21 days, with a typical range of 18 to 24 days in length $[1,2,3,4]$. The reproductive function of a cow or heifer is characterized by whether she displays normal estrous cycles or not. Many factors have potential influences on the presence or absence of estrous cycle of heifers and researchers already focused on it elaborately $[5,6,7,8,9,10,11,12]$. Body condition Score (BCS), Days after childbirth and or Postpartum Interval(PPI), parity number, ovarian characteristics, uterine blood flow, progesterone level(P4), climate and nutritional factors are mostly discovered influential factors in this arena [13, 14, 15, 16]. Moreover, the various species of heifers and different country's environmental condition could play vital roles in this case. However, discovering the presence of estrous cycle would definitely an important indication for reproductive management in the cow herd. This would help the herd management to synchronize the estrous and thus have more chance to make cow pregnant with the help of artificial insemination or even in a natural way [17, 18]. It is evident from [17] that, estrous synchronization protocol assists to get higher pregnancy rate in many countries (Fig. 1). However, finding out the presence of estrous cycle and pregnancy investigation in cattle usually done by ultrasound image analysis by an experienced inspector and some regular data analysis tools $[18,19,20,21,22]$. The usage of ultrasound image analysis for cattle is still very much subjective and expensive to some extent. Moreover, much experiences and skills are required for interpreting ultrasound image analysis to identify estrous cycle properly. 


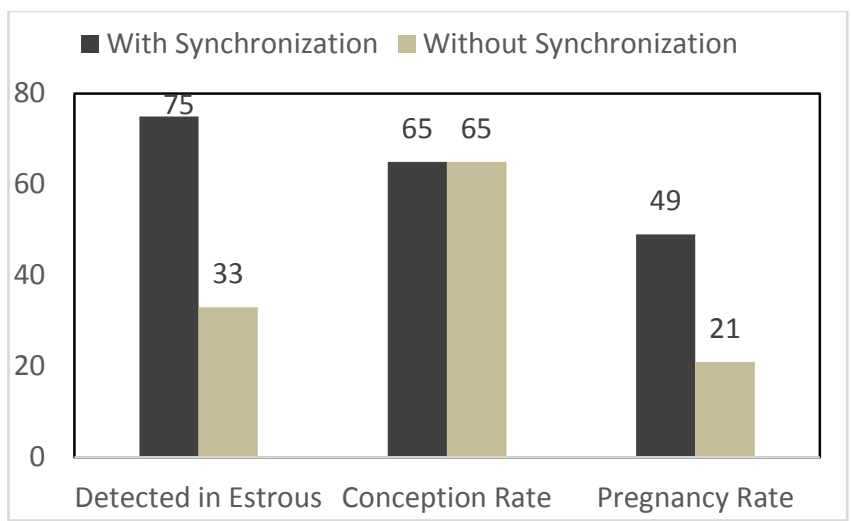

Fig. 1. Effect of Estrous Synchronization Protocol, adopted from [17]

Among the many factors of cattle productivity influence, the most influential one is the BCS, which is defined as "an effective management tool to estimate the energy reserves of a cow" $[13,23,24]$ and most widely used for herd management. There are many identified systems for measuring BCS, which varies according to different countries [13,23]. Using BCS to evaluate cattle does not require any special equipment and can be conducted anytime during the year. Poor body condition is associated with reduced income per cow, increased postpartum interval, increased dystocia, and lower weaning weight. The most common and widely used (USA and Japan) BCS scale ranges from 1 to 5 with 0.25 increments [24]. Though BCS measured subjectively and its reliability is questioned, it is also evident that BCS has relationship with many other factors of bovine, such as postpartum interval, parity, and etc. $[5,11,15,24]$. However, this investigation focused on three influential factors (BCS, postpartum interval, and parity) for understanding the presence and absence of estrous cycle using a new unique Bayesian Network Model (BNM). In total, 280 different Japanese Holstein cows observing with their BCS (2.0 to 3.25), postpartum interval and parity numbers to discover the ideal timing for artificial insemination to make them pregnant. It is also important to mention that, all these 280 samples found anestrus in their farm. The aim of this study is to find out the optimum factors to have an estrous cycle of bovine using Bayesian network model analysis in Japanese dairy industries. It is clear from National Livestock Breeding Center (NLBC, Japan) that, the overall conception rate of live beef and dairy cattle is decreasing in last 20 years in Japan (Fig. 2) [25]. Moreover, the findings of Bayesian network analysis could use for designing estrous synchronization protocol to improve cattle productivity and herd management. Moreover, using BNM analysis would assist the farm management to find out the presence of estrous cycle more objectively and in an accurate way.

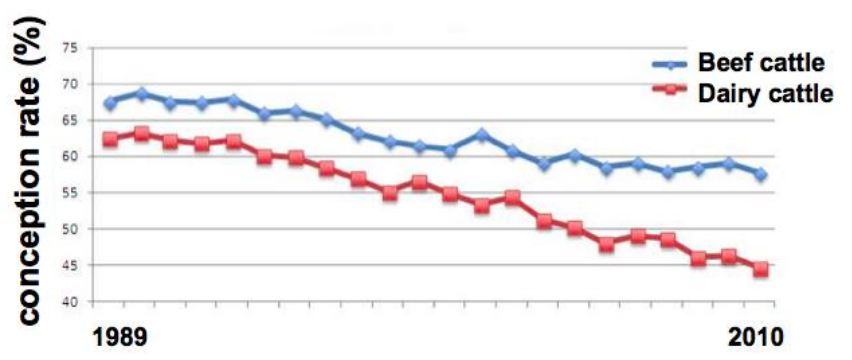

Fig. 2. The decreasing conception rate in Japan, partial adoption from NLBC, Japan

Using BNM for analyzing the presence or absence of estrous cycle is the most unique work in this arena. In addition, the Bayesian results deliver higher accuracy to find out the estrous cycle in relationships with BCS, Postpartum interval and number of parity. The rest of the paper is organized as follows, next the Bayesian network model section describes the proposed designed Bayesian network model for identifying estrous cycle using the data sets. Experiment section will introduce the overall experimental steps, data collection methods, and conditions in detail. The analytical results and its interpretation will present in Results and Discussion sub section. Finally, the paper concludes with future plans of this research and few challenges.

\section{IDENTIFYING ESTROUS CYCLE USING BAYESIAN NETWORK MODEL (BNM)}

Fig. 3 depicts the Bayesian network used in this investigation. Bayesian network is represented using the directed graph. The parent node indicates the cause, and the child node indicates the result. The proposed method uses three kind of information. Each node indicates the valuable such as BCS, (PPI)/Days after childbirth, Parity number, and Estrous cycle. The details are explained in the following fig. 3 .

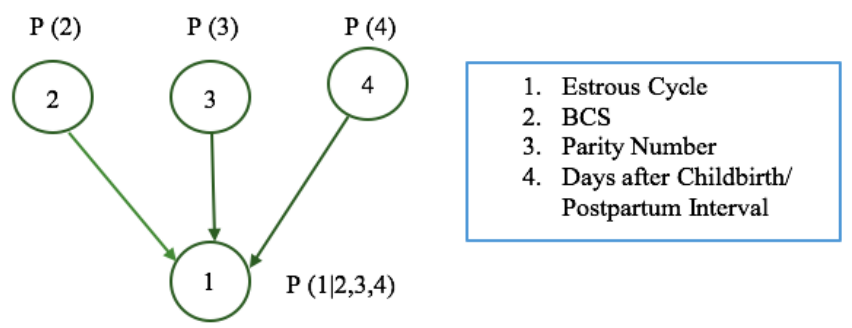

Fig. 3. The proposed Bayesian Network tree structure for identifying estrous cycle in Cattle

According to Bayes rules, posterior probability of having estrous cycle or not is designed with each individual parameter's prior and conditional probability. Here, The Bayes equation is used for each parameter as follows, 
$P($ estrous cycle $\mid B C S)=\frac{P(\text { estrous cycle }) P(B C S \mid \text { estrous cycle })}{P(B C S)}$

where, $P($ estrous cycle $)=$ Prior probability of having estrus or not; $P(B C S \mid$ estrous cyle $)=$ conditional probability of having estrus or not based on BCS information; $P(B C S)=$ probability of BCS evidence to find the posterior probability of having estrous cycle. Finally, the total posterior probability of the presence or absence of estrous cycle was determined under three evidences (BCS, PPI, Parity) by following (1).

\section{A. Body Condition Scoring (BCS)}

The research reveals to include BCS while considering the estrous cycle identification. The BCS is the most significant influential factors in bovine productivity. An organized process for determining BCS was created at the University of Pennsylvania to help achieve consistency and repeatability in BCS. This system finds its accuracy toward the mid-range scores (2.50 to 4.00), which includes most cattle in this investigation. This mid-range is the most critical for making farm management decisions and most influential for the farm nutritionist. The BCS outside this range indicate significant problems and varies significantly with respect to each individual inspector/observer. This research considering $\mathrm{BCS}_{4.0}$ methods (quarter-point increase) in 280 individual cattle of Morinaga Dairy Service Co. Ltd. (MDS), Japan and the following table describes the meaning of BCS scale. The $\mathrm{BCS}_{4.0}$ method ( 0.25 increase) have good repeatability across and within observers including simplified body scoring as well as have higher value as a diagnostic test [24]. The BCS process represents the observer's view into the certain anatomical sites for each cow's pelvic, loin areas, pin and hook bones, and etc. Next Table I briefly elaborates the observing BCS of 280 individual cows from the dairy farm of Iwate Prefecture, Japan under MDS cooperation.

TABLE I. BCS AND IT's GENERAL MEANING FOR 280 SAMPLE COW

\begin{tabular}{|l|l|}
\hline BCS & Meaning (in general) \\
\hline 2.25 & No fat pads on pin and hook bones- angular shape \\
\hline 2.5 & Palpable fat pads on pin and hook bones- angular shape \\
\hline 2.75 & $\begin{array}{l}\text { Pin bones- round shape and hook bones- angular shape } \\
\text { with less fat pads }\end{array}$ \\
\hline 3.0 & Fat pads on pin and hook bones- round shape \\
\hline 3.25 & Visible fat pads on pin and hook bones- round shape \\
\hline
\end{tabular}

\section{B. Postpartum Interval(PPI)/Days after Childbirth}

Each cow goes through a period of temporary infertility known as postpartum anestrus. Usually, cattle do not have estrous cycle during this period. The common term associated with this is postpartum interval (PPI), which is the duration from calving to the subsequent conception again. Several factors affect the postpartum interval of cows such as BCS, age and genetics. This research also includes PPI or days after calving parameter to evaluate the finding of estrous cycle in cows. It is also evident that BCS is affecting the PPI in beef and dairy cattle $[5,14,15,16,26]$. Therefore, this research introduces PPI as an individual parameter in the proposed BNM, which assists to find more accurate estimation of estrous cycle with sample data. The following table II describes and categories the PPI of 280 individual cows. In total, the proposed BNM consider 9 groups with one month (30 days) interval.

\section{Parity Number/Number of calves}

Parity is another important parameter in the proposed BNM. The total number of calves plays vital role in this investigation as it affects the probability of getting pregnant in the next subsequent time. The parity number is calculated without considering its first birth. In this investigation, among the 280 individual Holstein cattle, the highest number of parity for each cow is 9 and the lowest is 1 . Usually, the cow with higher parity might have less chance to resume their estrous cycle whereas the cow with parity range of 1 to 4 might have higher possibility to continue their estrous cycle on time.

TABLE II. DAYS AFTER CHILDBITH/PPI \& IT'S GROUP FOR BNM

\begin{tabular}{|l|l|}
\hline Days after Calving/PPI & Grouping \\
\hline $31-60$ days & 1 \\
\hline $61-90$ days & 2 \\
\hline $91-120$ days & 3 \\
\hline $121-150$ days & 4 \\
\hline $151-180$ days & 5 \\
\hline $181-210$ days & 6 \\
\hline $211-240$ days & 7 \\
\hline $241-270$ days & 8 \\
\hline$>271$ days & 9 \\
\hline
\end{tabular}

IV. EXPERIMENTAL SETUPS

To evaluate the finding of estrous cycle while considering BCS, PPI and Parity number, this research proposed a unique Bayesian Network Model (Fig.3). The overall experimental steps illustrate in the next Fig.4. Each individual cattle are identified with a unique number in the farm and then observed by an experienced inspector. The required BCS, PPI, Parity number and estrous cycle related data were collected. These sample data were then processed according to the proposed $\mathrm{BNM}$ to learn the system. The BCS measurement were carried out with well know $\mathrm{BCS}_{4.0}$ system and the PPI interval were categorized into 9 groups (Table I and II). Then, all these four individual parameters for each 280 individual data were feed into the proposed Bayesian model. The learning and validation of the model is described briefly in the Results and Discussion sub section.

\section{A. Conditions}

The followings are some of the important condition to mention during this research. All these 280 individual sample data were collected from the dairy farm of Iwate Prefecture with the cooperation of Morinaga Dairy Service (MDS) Co. Ltd., Japan. The BCS were observed in accordance with the UV method of Ferguson [24] by an experienced animal scientist of MDS. The PPI, Parity and other related information is collected from MDS. All of these 280 individual cattle were Japanese Holstein breed, which were found anestrus due to some health hazards in its own farm. The overall investigation for all these problematic dairy cow is under observation of MDS. The Bayonet 6 software, developed by AIST, Japan was used to design the proposed Bayesian network model. The final analytical results represented by using JMP data analytical tool, developed by SAS Institute Inc. 


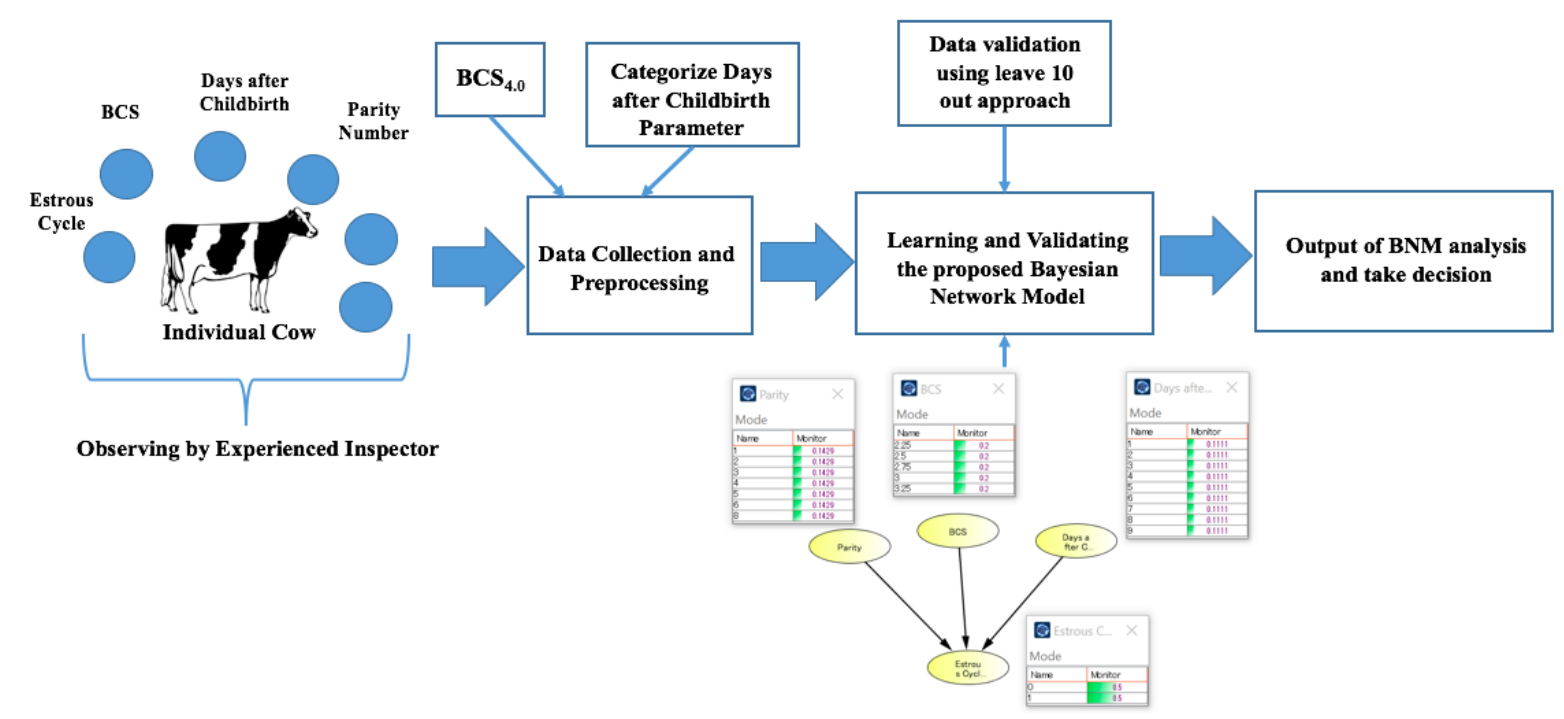

Fig. 4. The overall investigation methods using BNM

\section{B. Results and Discussions}

Bayesian network model assisted to visualize the changes of posterior probability as the evidence increases and thus assists to improve the accurate findings of estrous cycles with other methods. The approach of using BNM with the inclusion of BCS, PPI and parity parameters overlooked all previous estimation error of finding estrous cycle. The learning of Bayesian network includes 270 individual data and the rest of 10 data used for test purpose. The 10 data leave out approach improves the accuracy of the model and therefore 27 individual sets of learning and testing data sets used to validate the proposed model. Moreover, when the proposed model acquired higher confidence (less entropy value), the estimation accuracy for the presence of estrous cycle lies in between 93 to $100 \%$.

There were 270 samples used to learn the model and the learning is based on Greedy search algorithm. As a measurement criterion for the appropriateness of a graph structure, information criteria AIC is used. The Bayesian tree was formed by using the estimation of BCS, PPI and Parity number of each cow. Finally, the posterior probability was calculated under these three evidence by following (1). The overall optimum factors for the presence of estrous cycle according to BCS are illustrated in next table III. The fact of the table is the general output of the proposed BNM with learning datasets.

It is evident from many researches that, BCS plays most vital role for affecting all other individual parameters. Therefore, the table III focused only on BCS mostly. The posterior probability for the presence of estrous cycle according to BCS, PPI (1 to 4 groups) and Parity (1 to 4 ) is measured with 270 sample data. When the BCS is 2.75 , the PPI is 31-60 days (group 1) and the parity is 1, the probability of having estrous cycle is $80 \%$. At the same time, when the BCS is 2.5, PPI is 91-120 days (group 2) and Parity is 3, the probability of the presence of estrous cycle is same $(80 \%)$. The benefit of using Bayesian model is to find out easily these kind of many more relationships in the productivity management. It is now also clear from the table that, BCS is actually not only the significant factor affecting the presence or absence of timely estrous cycle in cattle. Next, the analysis of BNM with 27 individual test datasets is shown in fig. 5 .

The overall highest estimation accuracy for finding the presence of estrous cycle based on proposed BNM is $93 \%$ and the average accuracy for all data set is almost $55 \%$ and lowest average accuracy is $50 \%$, while the log-likelihood is more than 0.7 . Using the suspended rule on average likelihood, this research discovered the average estimation accuracy of finding estrous cycle, which represents in next fig. 6.

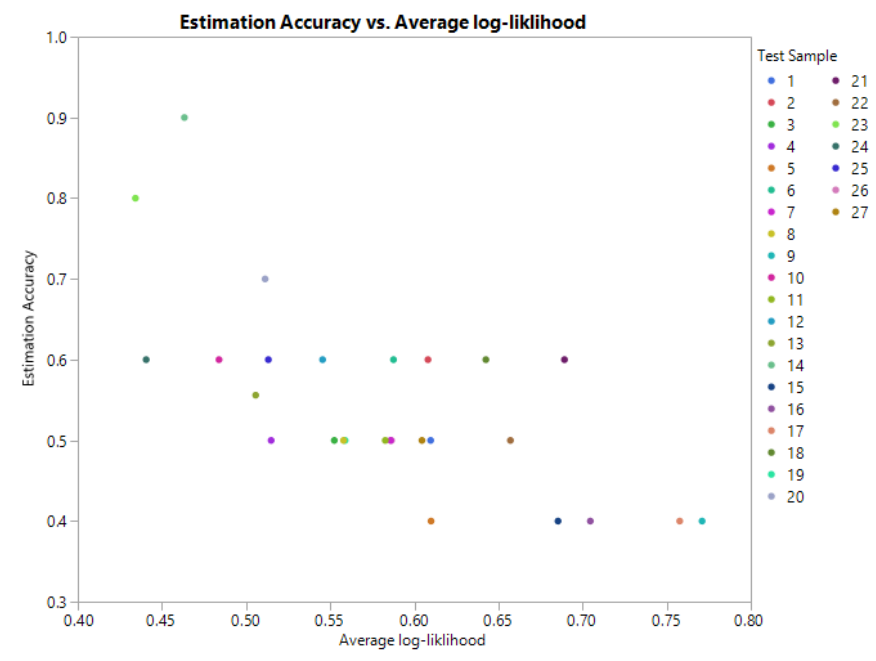

Fig. 5. Estimation accuracy of individual data sets

It is clear from fig. 6 that, when the likelihood is $<0.5$, the highest average estimation accuracy for the presence of estrous cycle is almost $73 \%$ (total 40 cattle) and lowest is $50 \%$ when the likelihood is considering $<0.7$. On the contrary, most of the dairy cows lies within the likelihood of $<0.6(130+40$ $=170$ cattle) and the average estimation accuracy for considering all cattle is 55\%. However, the research 
discovered that, the sample data might not be enough to satisfy the proposed Bayesian model. Therefore, the entropy of proposed model's outputs was calculated to achieve reliable discrimination and use it for discrimination-suspension rule [27]. Entropy indicates or interprets as the risk of incorrect discrimination and if entropy exceeds some predefined discrimination threshold, then the discrimination could be suspended. The following equation used to calculate the entropy between two states of estrous cycle in the designed model.

$$
\text { entropy }=-\sum_{i=1}^{2} P_{i} \log P_{i}
$$

where, $P_{i}=$ results of posterior probability for the presence (1) or absence (0) of estrous cycle. The higher entropy means the designed network model is ambiguous and less entropy derive the more confident model.

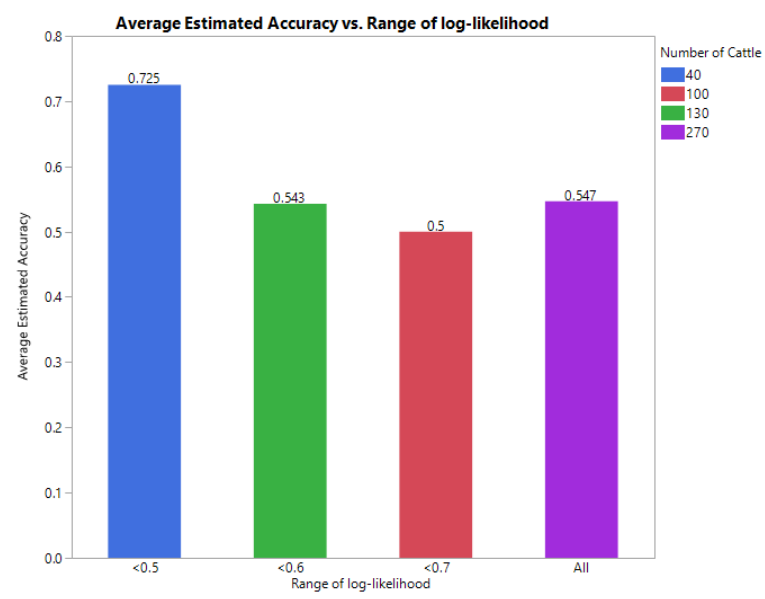

Fig. 6. Average accuracy vs suspended log likelihood based on total number of cattle

TABLE III. The Probability of Having Estrous CyCle IN 280 Cows ACCORdING To BCs, PPI \& PARITY

\begin{tabular}{|c|c|c|c|}
\hline BCS & PPI (according to group) & Parity (parity 1 to 4) & Presence of Estrous cycle (\%) \\
\hline \multirow{4}{*}{2.25} & 1 (31-60 days) & 1 & 13 \\
\hline & 2 (61-90 days) & 2 & 20 \\
\hline & 3 (91-120 days) & 3 & 60 \\
\hline & 4 (121-150 days) & 4 & 50 \\
\hline \multirow{4}{*}{2.5} & 1 (31-60 days) & 1 & 44 \\
\hline & 2 (61-90 days) & 2 & 65 \\
\hline & 3 (91-120 days) & 3 & 80 \\
\hline & 4 (121-150 days) & 4 & 75 \\
\hline \multirow{4}{*}{2.75} & 1 (31-60 days) & 1 & 80 \\
\hline & 2 (61-90 days) & 2 & 71 \\
\hline & 3 (91-120 days) & 3 & 71 \\
\hline & 4 (121-150 days) & 4 & 50 \\
\hline \multirow{4}{*}{3.0} & 1 (31-60 days) & 1 & 50 \\
\hline & 2 (61-90 days) & 2 & 75 \\
\hline & 3 (91-120 days) & 3 & 67 \\
\hline & 4 (121-150 days) & 4 & 33 \\
\hline \multirow{4}{*}{3.25} & 1 (31-60 days) & 1 & 60 \\
\hline & 2 (61-90 days) & 2 & 50 \\
\hline & 3 (91-120 days) & 3 & 50 \\
\hline & 4 (121-150 days) & 4 & 50 \\
\hline
\end{tabular}

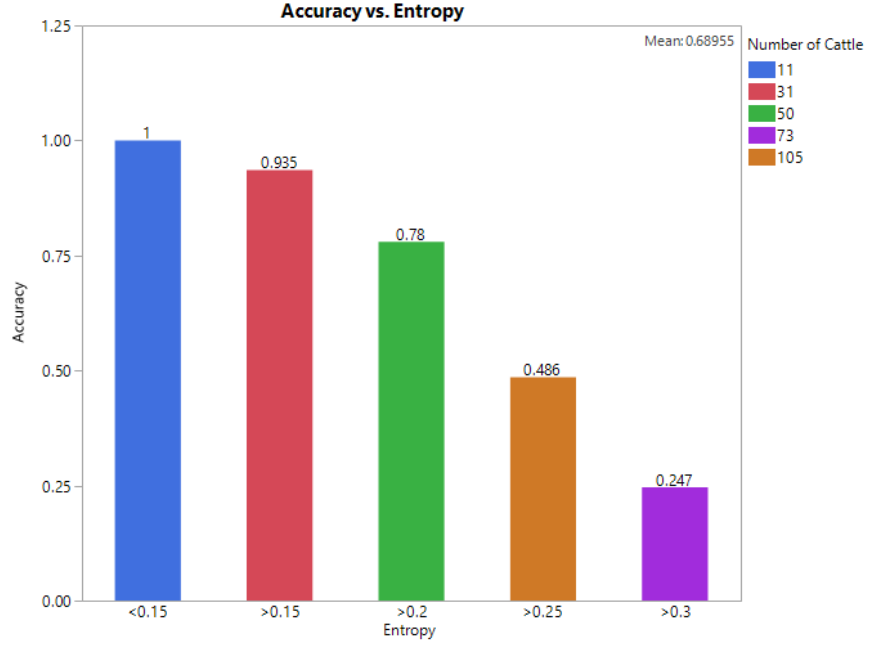

Fig. 7. The estimation accuracy of proposed BNM with higher confidence
According to entropy calculation, when the proposed model gets higher confidence $(0.15<$ entropy>0.15), the accuracy rate lies in between $93 \%$ to $100 \%$, which is one of the most significant finding in this research. Fig. 7 represents the results of the proposed BNM with higher confidence. In addition, Fig. 8 illustrates briefly the most influential parameters distribution according to the accuracy estimation with high confident model. These distributions would assist the farm management to find out most optimum cattle to have estrous cycle on due course.

All of these findings represent significant reliability and confidence to estimate the presence of estrous cycle with comparison to other traditional methods. In addition, all these sample cattle were previously anestrus due to some health hazards and other reasons in their farm. This investigation method could easily deploy to other healthy cattle for designing proper estrous synchronization protocol too. Moreover, the analytical approach of using Bayesian network discovers the most optimum conditions for each individual 
cattle to find the presence of estrous cycle. The objective estimation of finding out the presence or absence of estrous cycle in cattle definitely boosts up the productivity in this arena and as well as leads to a new field of research.

\section{CONCLUSION AND FUTURE PLAN}

This research presented the discovering method of cattle's estrous cycle presence using a new approach of Bayesian network model. The inclusion of body condition parameters, postpartum intervals and parity in the model helped to evaluate more accurate objective estimation of estrous cycle presence in cattle. The results and analysis confirmed that, the more accurate and optimum factors for cattle productivity could be found by the proposed BNM. The authors believe, the objective estimation definitely provides boost-up in the
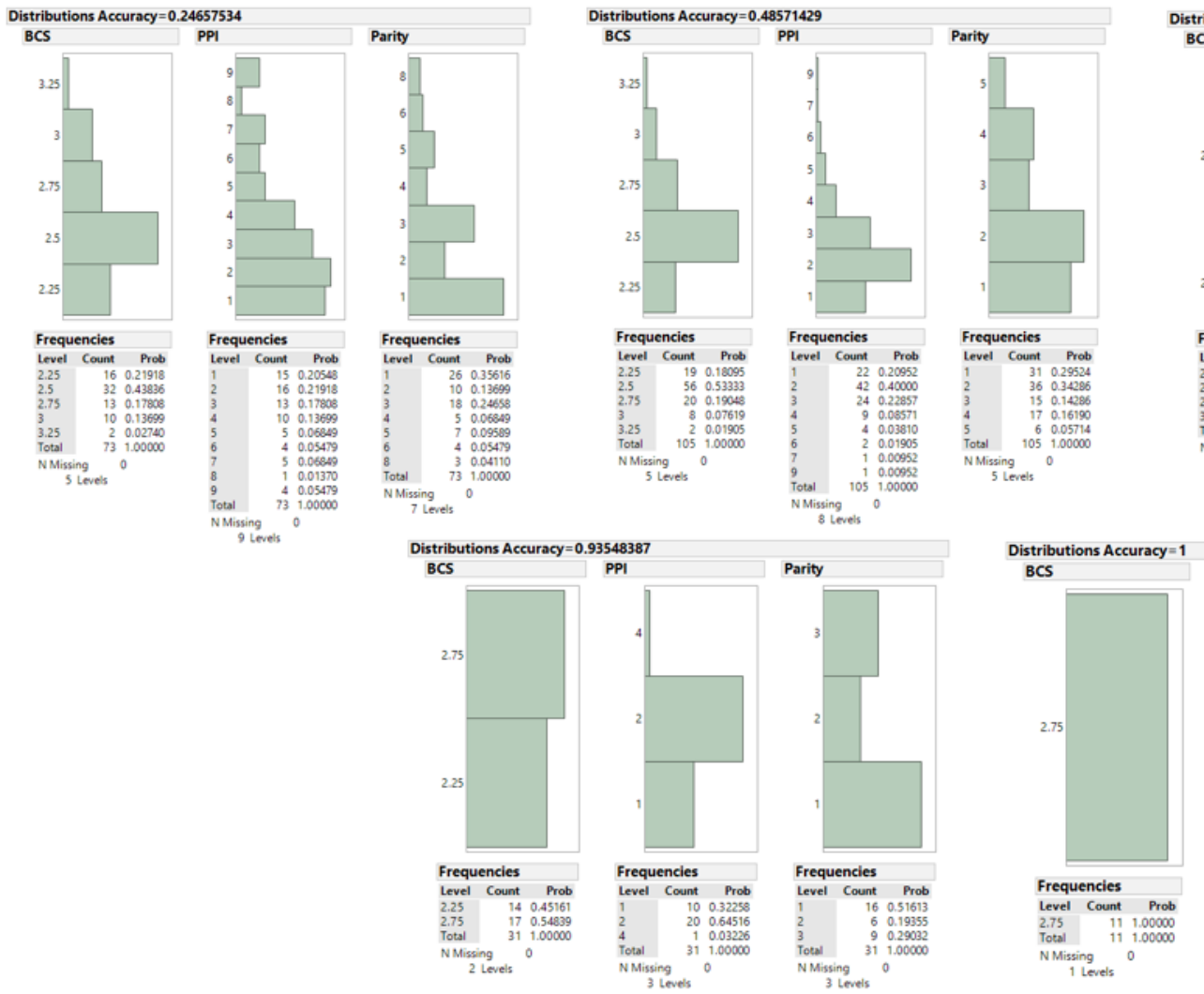

productivity of livestock industry in Japan and others countries. In future, the authors would like to include other parameters of cattle for finding out the presence of estrous cycle. In addition, the proposed model could be validated by using more sample datasets in future. Therefore, the proposed methods would get higher confidence and reliability to use at industry level.

\section{ACKNOWLEDGMENT}

This investigation is funded and supported by Ministry of Agriculture, Forestry and Fisheries (MAFF), Japan. The authors also show gratitude and appreciation to the Morinaga Dairy Service Co. Ltd., Japan for their constant support during this investigation.

Fig. 8. Distribution of BCS, PPI and Parity according to high confident model for estimating the presence or absence of estrous cycle

\section{REFERENCES}

[1] J. C. Whittler, "Reproductive Anatomy and Physiology of the Cow", Department of Animal Sciences. University of Missouri, Accessed December, 2015.

[2] P. D. Burns, "The Dairy Cow Heat Cycle", Colorado State University, Accessed December, 2015.

[3] J. A. Parish, J. E. Larson, and R. C. Vann, "The Estrous cycle of Cattle", Mississippi State University in cooperation with US Department of Agriculture, Publication No.2616, 2010.

[4] G. Perry, "The Bovine Estrous Cycle- FS921A", South Dakota State University-Cooperative Extensive Service USDA, Accessed December, 2015.

[5] J. Walker, and G. Perry, "Cow Condition and Reproductive Performance", Proceeding of The Range Beef Cow Symposium XX, Colorado, USA, December, 2007.

[6] L. F. M Pfeifer, S.C.B.S. Leal, A. Scheneider, E. Schemitt, and M.N. Correa, "Effect of ovulatory follicle diameter and progesterone concentration on the pregnancy rate of fixed time inseminated lactating beef cows", Revista Brasileria de Zootecnia, Vol. 41, No. 4, 2012, pp. 1004-1008.

[7] M. Matsui, and A. Miyamoto, "Evaluation of ovarian blood flow by colour Doppler Ultrasound: Practical use for reproductive management in the cow", The Veterinary Journal, 181, 2009, pp.232-240.

[8] T.A.Zacarias, S.B. Sena-Natto, A.S. Mendonca, M.M. Franco, and R.A. Figueiredo, "Ovarian Follicular Dynamics in 2 to 3 months old Nelore Calves (Bos Taurus indices)", Journal of Animal Reproduction, Vol. 12, No.2, June,2015, pp.305-311.

[9] G.A. Perry, M.F. Smith, A.J. Roberts, M.D. MacNeil, and T.W. Geary, "Relationship between size of the ovulatory follicle and pregnancy success in beef heifers", Journal of Animal Science, 85:684-689, 2007.

[10] A. Honnens, C. Voss, K. Herzog, H. Niemann, D. Rath, and H. Bollwein, "Uterine blood flow during the first 3 weeks of pregnancy in Dairy Cows" Journal of Theriogenology, Vol.70, 2008. Pp.1048-1056. 
[11] G. Campanile, G. Neglia, R. Di Palo, B. Gasparrini, C. Pacelli, M. D'Occhio, and L. Zicarelli, "Relationship of body condition score and blood urea and ammonia to pregnancy in Italian Mediterranean buffaloes", Reproduction Nutrition Development, EDP Sciences, 2006, 46 (1), pp.57-62.

[12] G. A. Perry, O. L. Swanson, E. L. Larimore, B. L. Perry, G. D. Djira, and R. A. Cushman, "Relationship of follicle size and concentrations of estradiol among cows exhibiting or not exhibiting estrus during a fixedtime AI protocol", Journal of Domestic Animal Endocrinology, 48(2014), pp.15-20.

[13] W. Kellogg, "Body Condition Scoring with dairy cattle- FAS4008", University of Arkansas, USA, Accessed on: January 2016.

[14] J.M. Bewley, and M.M. Schutz, "Review: An interdisciplinary review of Body Condition Scoring for Dairy Cattle", The Professional Animal Scientist 24(2008), pp. 507-529.

[15] F.C. Castro, J.O. Porcayo, R.J. Ake-Lopez, J.G.M. Monforte, R.C. Montes-Perez, and J.C.S. Correa, "Effect of Body Condition Score on Estrous and Ovarian function characteristics of Synchronized BeefMaster Cows", Journal of Tropical and Subtropical Agroecosystems, 16(2013), pp.193-199.

[16] K. Yamada, T. Nakao, and N. Isobe, "Effects of Body Condition Score in Cows Peripartum on the onset of the Postpartum Ovarian Cyclicity and Conception rates after Ovulation Synchronized/ Fixed-Time Artificial Insemination", Journal of Reproduction and Development, Vol. 49, No. 5, 2003, pp.381-388.

[17] M. DeJarnette, "Estrus Synchronization: A Reproductive Management Tool", White Paper, Select Sires Inc., Ohio, USA, 2004.

[18] M. Takagi, N. Yamagishi, I.H. Lee, K. Oboshi, M. Tsuno, and M.P.B. Wijayagunawardane, "Reproductive management with Ultrasound Scanner Monitoring System for a high-yielding Commercial Diary Herd Reared under Stanchion Management Style", Asian-Australian Journal of Animal Science, 2005, Vol. 18, No. 7, pp. 949-956.

[19] G.P. Adams, and J. Singh, "Bovine Bodyworks: ultrasound Imaging of Reproductive Events in Cows", WCDS Advances in Dairy Technology, Vol. 23, 2011, pp. 239-254.

[20] J.H.M. Viana, E.K.N. Arashiro, L.G.B. Siqueira, A.M. Ghetti, V.S. Areas, C.R.B. Guimaraes, M.P. Palhao, L.S.A. Camargo, and C.A.C Fernandes, "Doppler Ultrasonography as a tool for Ovarian Management", Journal of Animal Reproduction, Vol. 10, No. 3, September 2013, pp. 215-222.

[21] P.M. Fricke, and G.C. Lamb, "Practical applications of ultrasound for reproductive management of beef and diary cattle", Proceedings of The Applied Reproductive Strategies in Beef Cattle Workshop, Kansas, USA, September 2002.

[22] G.C. Lamb, C.R. Dahlen, and D.R. Brown, "Reproductive Ultrasonography for monitoring Ovarian Structure Development, Fetal Development, Embryo Survival and Twins in Beef Cows", The Professional Animal Scientist Symposium, No. 19, 2003, pp. 135-143.

[23] Anonymous, "Body Condition Scoring in Dairy Cattle- AI10782", White Paper, Elanco Animal Health, 1-800-428-4441, 2009.

[24] J.D. Ferguson, D.T. Galligan, and N. Thousen, "Principal Descriptor of Body Condition Score in Holstein Cows", Journal of Dairy Science, No.77, 1994, pp.2695-2703.

[25] Report of National Livestock Breeding Center, Japan. Website: http://www.nlbc.go.jp/en/, Accessed January, 2016.
[26] W.J. Burkholder, "Use of Body Condition Scores in Clinical Assessment of the Provision of the Optimal Nutrition", JAVMA, Vol.217, No. 5, September 2000.

[27] O.Fukuda, T.Tsuji, and M.Kaneko, "A human supporting manipulator based on manual control using EMG signal", Journal of the Robotics Society, Japan, Vol.18, No.3, 2000, pp.79-86.

\section{AUTHORS PROFILE}

Kohei Arai, He received BS, MS and PhD degrees in 1972, 1974 and 1982, respectively. He was with The Institute for Industrial Science and Technology of the University of Tokyo from April 1974 to December 1978 also was with National Space Development Agency of Japan from January, 1979 to March, 1990. During from 1985 to 1987, he was with Canada Centre for Remote Sensing as a Post Doctoral Fellow of National Science and Engineering Research Council of Canada. He moved to Saga University as a Professor in Department of Information Science on April 1990. He was a councilor for the Aeronautics and Space related to the Technology Committee of the Ministry of Science and Technology during from 1998 to 2000. He was a councilor of Saga University for 2002 and 2003. He also was an executive councilor for the Remote Sensing Society of Japan for 2003 to 2005 . He is an Adjunct Professor of University of Arizona, USA since 1998. He also is Vice Chairman of the Commission "A" of ICSU/COSPAR since 2008. He wrote 33 books and published 510 journal papers. He is now Editor-in-Chief of IJACSA and IJISA.

Osamu Fukuda received his B.E. degree in mechanical engineering from Kyushu Institute of Technology, lizuka, Japan, in 1993 and the M.E. and Ph.D. degrees in information engineering from Hiroshima University, Japan in 1997 and 2000, respectively. From 1997 to 1999, he was a Research Fellow of the Japan Society for the Promotion of Science. He joined Mechanical Engineering Laboratory, Agency of Industrial Science and Technology, Ministry of International Trade and Industry, Japan, in 2000. Then, he was a member of National Institute of Advanced Industrial Science and Technology, Japan from 2001 to 2013. Since 2014, he has been a Professor of Graduate School of Science and Engineering at Saga University, Japan. Prof. Fukuda won the K. S. Fu Memorial Best Transactions Paper Award of the IEEE Robotics and Automation Society in 2003. His main research interests are in human interface and neural networks. Also, he is currently a guest researcher of National Institute of Advanced Industrial Science and Technology, Japan. Prof. Fukuda is a member of IEEE and the Society of Instrument and Control Engineers in Japan.

Iqbal Ahmed got his Bachelor of Science (BSc) Honors degree in Computer Science and Engineering from University of Chittagong, Bangladesh in 2007 and achieved joint Master degree from PERCCOM program of European Union in September 2015. He received his Master of Complex System Engineering degree from University of Lorraine (UL), France then Master in Technology from Lappeenranta University of Technology (LUT), Finland and Master degree in Pervasive Computing and Communication for Sustainable development from Lulea University of Technology (LTU), Sweden. Since October 2015, he is enrolled as a doctoral student in the Department of Information Science, Saga University, Japan. In profession, he worked in the Department of Computer Science and Engineering, University of Chittagong, Bangladesh as an Assistant professor since February 2011. He has been awarded Cat-A scholarship of Erasmus Mundus from European Union two times in 2010 and 2013 respectively. His current research interest lies in the field of green and sustainable computing and information processing. 